\title{
Article \\ Application of Carbon Nano-sensors in 3-D Braided Composite Material Damage Detection
}

\author{
Qingxia Dong ${ }^{1 *}$, Gang Ding ${ }^{2}$ and Jie Cheng ${ }^{3}$ \\ 1 School of Computer Science and software, Tianjin Polytechnic University; dongqingxia@tjpu.edu.cn \\ 2 Department of The Management and Construction of Teaching Resources Tianjin Radio and TV University; \\ Dingg@tjrtvu.edu.cn \\ 3 Center of informatization, Tianjin Polytechnic University; chengjie@tjpu.edu.cn \\ * Correspondence: dongqingxia@tjpu.edu.cn; Tel.: +86-156-2032-5011
}

\begin{abstract}
Aiming at the damage detection and continuous monitoring of high efficiency composite structure, the method of carbon nano sensor embeded in the 3-D braided composite material specimen was put forward and the method of damage location for 3-D braided composite material was described. In this paper, the mechanical and electrical characteristics of carbon nano-fiber were analyzed. Considering the massive data of the health monitoring of composite processing, to ensure that the system has good real-time performance, quaternary matrix Singular Value Decomposition algorithm is proposed to simplify data and improve the efficiency of the algorithm. The existence of injury is determined and the damage location is calculated by comparing with key features of healthy specimen. Experimental results prove the method is feasible and effective.
\end{abstract}

Keywords: carbon nano-fibers; 3-D braided composite material; damage detection; SVD

\section{Introduction}

As a kind of engineering material, 3-D braided composite has been widely used in aerospace and other high-tech industries due to its high specific stiffness, good corrosion resistance, good fatigue resistance, good design ability and other excellent properties. As the main bearing part, composites can be affected by adverse factors such as high temperature, high pressure, corrosion, aging of materials, and high altitude environment, etc. Therefore, the material can be fatigued and damaged under the combined action of various complicated loads. As the main load-bearing materials of aerospace, a complete Health Monitoring System[1] must be established under its working condition to monitor the local damage in real time and give a quantitative assessment. So accurate information for damage detection of materials and structures can be provided, and huge losses caused by composite materials structural damage are avoided. It is hard to find out the internal damage by visual inspection or beating. The common nondestructive testing methods for composite materials are visual damage, ultrasonic testing, X-ray inspection, acoustic emission testing, eddy current, rays, fiber Bragg grating[2]. Because of the particularity of composite structure, these methods have many disadvantages, such as long detection time, low precision and complex detection process, the most important is the methods above can not monitor the structure health condition continuously. All these make them inconvenient to use. Bragg grating sensor has low cost, no electromagnetic interference, can monitor internal structure change etc, but optical fiber sensor is brittle, poor knitting feasibility and uneasy to be connected. All of this illustrates that optical fiber sensor is difficult to handle. In this article carbon nano-sensor is used as detecting means[3]. Health monitoring of 3-D braided composites strain detection and damage identification technology are researched. SVD is used to reduce the dimensions of large data matrix 
and extract feature, as a result the amount of data processing is cut down and the system's real-time performance is enhanced. The simulation results show that the method is feasible in composite damage detection.

\subsection{Carbon Nanotubes}

Carbon nanotubes, also known as buckytubes, are made of a sheet of graphene that is rolled into a cylinder. There are two forms of carbon nanotubes (Single-wall carbon nanotubes (SWNT) and multi-wall carbon nanotubes (MWNT)) that can exhibit high structural perfection. SWNT are composed of one of these sheets, while MWNT are made of multiple graphite sheets rolled into individual sheets and stacked concentrically.

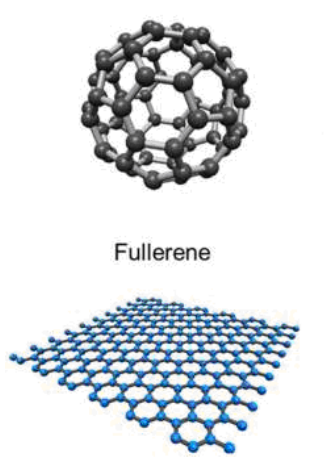

Graphene
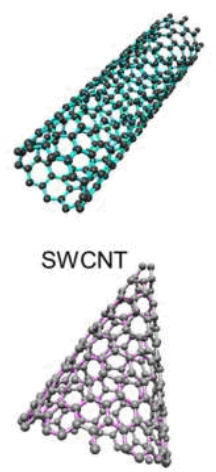

Nano cone
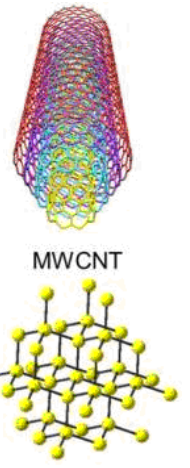

Nano diamond

Figure 1. Single-walled, double-walled and multi-walled carbon nanotubes HRTEM image.

As one dimensional nano materials, carbon nanotubes have light weight and perfect hexagonal structure, which has many abnormal mechanical, electrical and chemical properties. As shown in Table 1, SWNT with small diameters have both a high Young's Modulus and high tensile strength. The strength of carbon nanotubes can reach 300 times than steel, while the density of it is only $1 / 6$ of the steel[6].

Table 1. Comparison of Mechanical Properties and Thermal Conductivity of Individual Carbon SWNT, MWNT, Steel.

\begin{tabular}{cccc}
\hline Material & Modulus(GPa) & Tensile Strength(GPa) & Thermal Cond. $\left(\mathbf{W} /\left(\mathbf{m}^{*} \mathbf{K}\right)\right)$ \\
\hline SWNT & 600 & 30 & 2000 \\
MWNT & 1200 & 55 & 3800 \\
Steel & 210 & 1.5 & 50 \\
\hline
\end{tabular}

\subsection{Carbon Nano-fibers}

Carbon nano-fibers can be produced by the dry spinning process from CNT arrays. According to its structural characteristics, it can be divided into hollow carbon nano-fiber and solid carbon nano-fiber. These two kinds both have specific strength, specific modulus, light weight, good thermal conductivity. But beyond that carbon nano-fibers also have many advantages, such as few defects, large length-diameter ratio, large specific surface area, compact structure and so on. Their diameter is generally in $10 \mathrm{~nm} 500 \mathrm{~nm}$ [7]. It is a new generation of special fiber materials between carbon nanotubes and carbon fiber. It is a kind of high performance fiber, which has the inherent characteristic of carbon material and the softness and processability of the textile fiber. It has been widely used in aerospace, transportation, sports and leisure products, medical, mechanical, textile and other fields. Because the 3-D braided composite has certain conductivity, and the diameter of the carbon nano-fibers is several tens of microns, so carbon nano-fibers cannot be woven directly with composite materials. 
The method is to make carbon nano-fibers sensor. By adding protective layer to the outer carbon nano-fibers to make it become insulating, increase its diameter and improve its knitting feasibility.

\subsection{Characteristics of carbon nano-fibers}

Carbon nano-fibers show great multifunctional properties; they can be embedded in composites; serving as both strain sensors and load-carrying filaments. Carbon nano-fibers made by dry spinning shows good mechanical and electrical properties after twisting and winding technology. The stress-strain curve of the Carbon nano-fibers can be described in detail in a three region[8]. The increments in carbon nano-fibers strain resistance (DR/R) (i.e. strain sensitivity or strain coefficient) are measured between 0.4 and 0.5 . The fibers production rate is about $30 \mathrm{~m} / \mathrm{min}$.
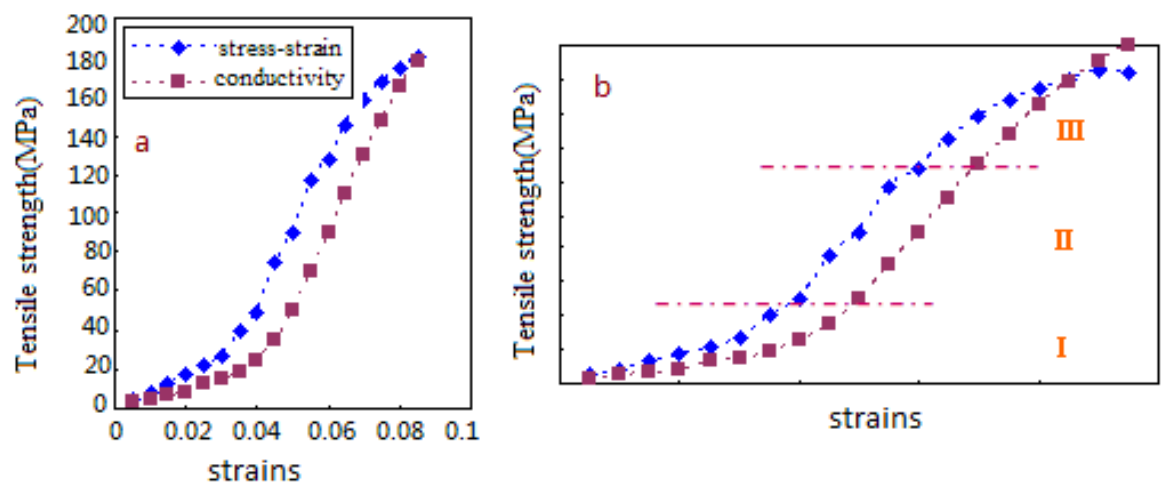

Figure 2. Mechanical and electrical properties of carbon nano-fibers (a) and its characteristic change in the three regions (b).

The results of tensile test are shown in Figure 2. The characteristic of the'S'shape stress - strain curve (blue) can be presented by the gradient of the three zones. The curve starts at the I area with a slow slope, and the slope is kept constant with the slow increase of strain into the area II. In the III zone, the stress strain curve is obviously attenuated until the specimen is broken. The red curves shows that $\mathrm{dR} / \mathrm{R}$ changes with the pressure, these data reflect the different gradient of the three regions; it can be seen in the area III slope is significantly increased relative to the II area. All above are shown in chart (b). This work improves the understanding of the interplay relationship between the mechanical and electromechanical behavior, which can contribute to enhanced CNT fiber sensor technology. At room temperature, the study of the carbon nano-fibers has a conductivity of $315 \mathrm{~S} / \mathrm{cm}$, which is higher than that of a single strand of fibers reported by Zhang et al. The conductivity of carbon nano-fibers is affected by temperature, in the metal, the conductivity decreased with temperature increasing; in the fiber, it increases with temperature, and gradually decreased at low temperatures (see Figure 3 (b)), which is the typical behavior of the fiber. 

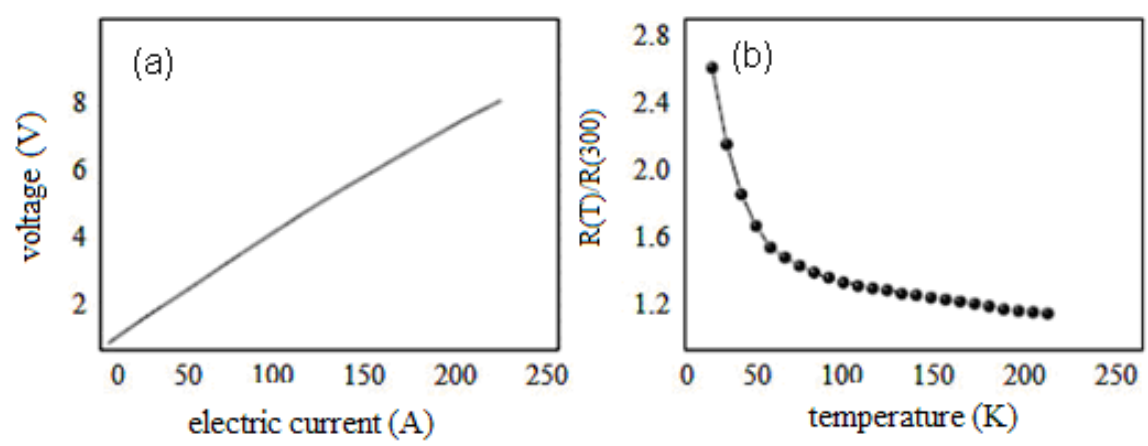

Figure 3. Electrical conductivity of carbon nano-fibers.

\subsection{Characteristics of carbon nano-sensors}

The structure of carbon nano-fibers sensor used in the health monitoring system is shown in Figure 4. It is composed of 1 carbon nano-fibers, 1 insulating protective layer, 2 line connector, These sensors are embed in the specimen as an array.

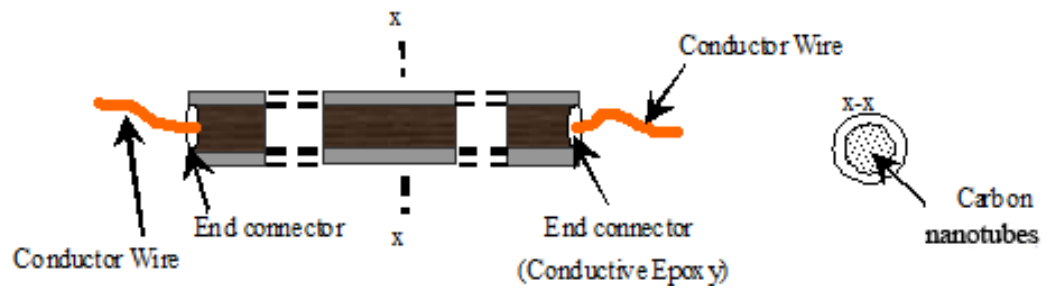

Figure 4. Carbon nano-fibers sensor structure.

The resistance of carbon nano-fibers increases monotonically with pressure. There are two factors influencing the resistance in tensile force, one is the pressure on individual carbon nanotubes; another is the radial force in carbon nano-fibers make contacts between adjacent carbon nanotubes have increased. The latter is clearly not the cause of the increase in resistance, as increasing contact causes resistance to decrease. Thus, the increase of resistance with pressure is caused by the compression of individual carbon nanotubes.

According to Ohm's law:

$$
R=\frac{\rho L}{A}
$$

In the formula (1) R is the impedance, is resistivity, $\mathrm{L}$ is length of CNFs and A is cross-sectional area of CNFs. The change in resistance of carbon nano-fibers can be calculated by formula (2).

$$
\frac{\Delta R}{R}=\frac{\Delta \rho}{\rho}+\frac{\Delta L}{L}-\frac{\Delta A}{A}
$$

The change in length of carbon nano-fibers can be described as

$$
\frac{\Delta L}{L}=\varepsilon_{11}-\frac{\varepsilon_{11}^{2}}{2}
$$

If carbon nano-fibers deformation is smaller, the second term is negligible. Since the Poisson effect, the cross-section changes of carbon nano-fibers is transversely isotropic, cross section of sensor can be expressed as

$$
\dot{A}=A\left(1-2 v_{12} \varepsilon_{11}\right)
$$


In the formula (4) is Poisson's ratio of CNFs. So

$$
\begin{gathered}
\frac{\Delta A}{A}=-2 v_{12} \varepsilon_{11} \\
\frac{\Delta R}{R}=\frac{\Delta \rho}{\rho}+\varepsilon_{11}+2 v_{12} \varepsilon_{11}=\frac{\Delta \rho}{\rho}+\varepsilon_{11}\left(1+2 v_{12}\right)
\end{gathered}
$$

There are two reasons for the change in resistance. One is the strain produced in the system; the second is the rate of change in resistance. Because the load-bearing and relative resistivity increment of the material have good symmetry, so the carbon nano-fibers sensor has better linearity within a certain range of bearing.

\section{3-Dcomposite materials embedded in carbon nano-fibers}

\subsection{3-D braiding technology}

In order to monitor the structural health of 3-D braided composite specimens, carbon nano-fibers sensors and carbon fibers are woven together. The specimens are all woven by 4-Step 3-D braided technology. In each machine cycle, the movement of yarn carrier include four steps: first step[12], The adjacent row carrier have one unit alternating motion along the $X$ direction; second step, the adjacent column carriers have a unit of alternating motion along the $Y$; third step, the adjacent row carrier moving relative to each other in the opposite direction with the first step; fourth step, adjacent column carriers relative motion, contrary to the second step direction. When a programmed cycle is completed, the arrangment of yarn carriers returns to the initial position. In this process, the yarn carriers Tighten the yarn along $\mathrm{Z}$ direction (specimen forming direction) to make the yarn closely intertwined.

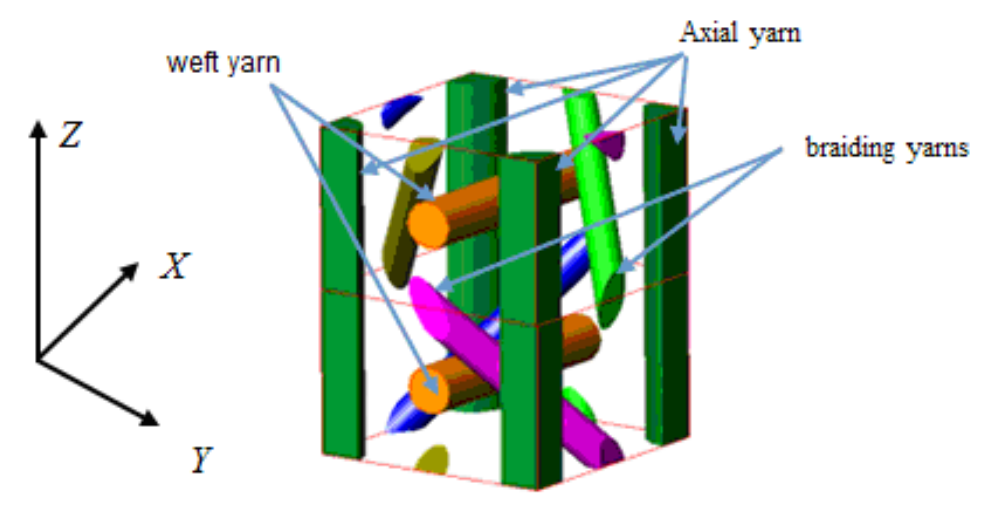

Figure 5. Three dimensional six dimensional fabric structure.

In experiment specimens carbon nano-sensors are embedded in composite material as axial yarns and sixth-directional yarns by 4-Step 3-D braiding technology. Figure.5 shows the structure of 3-D six dimensional fabric. The sixth-directional yarns (carbon nano sensors) are added in according to the optimal proportion calculated beforehand during weaving process, and they are arranged at intervals along the row direction.

\subsection{Carbon nano-fiber sensors array}

In Figure 6, the orientation of the carbon nano-fiber sensors embedded in the 3-D six direction braided preform is presented, which is the axial yarn and the sixth direction perpendicular to the fabric forming direction. Thus, the carbon nano-fibers form a sensor array in the interior of the preform. In the sensor array, the row and column sensors are connected by conducting wires, and the detection 
and location of internal damage can be achieved by sampling, processing and analyzing the signal of the row and column.

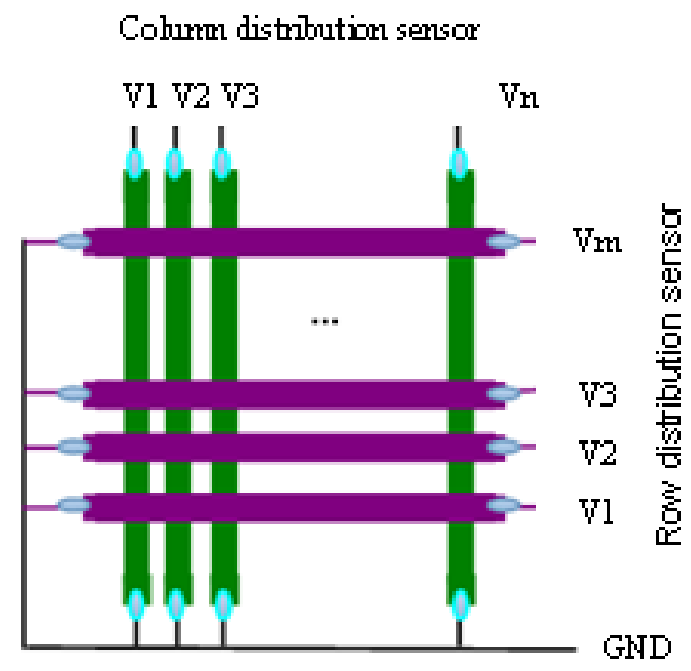

Figure 6. Diagram of the sensor array embedded in the preform.

In actual program, the density and quantity of carbon nano-fibers embedded in different specimens are different. They are composed of $m^{*} n$ matrix shapes (look like Figure 6). If the test object is larger, such as an aircraft wing, wind turbine blade, the mechanical analysis should be made on the object first, the carbon nano-fibers will be arranged in the easily damaged parts to avoid dense sensor layout and improve the detection cost.

\section{Experiments}

The structural damage detection system of 3-D braided composites based on carbon nano-sensors is shown in Figure 7.

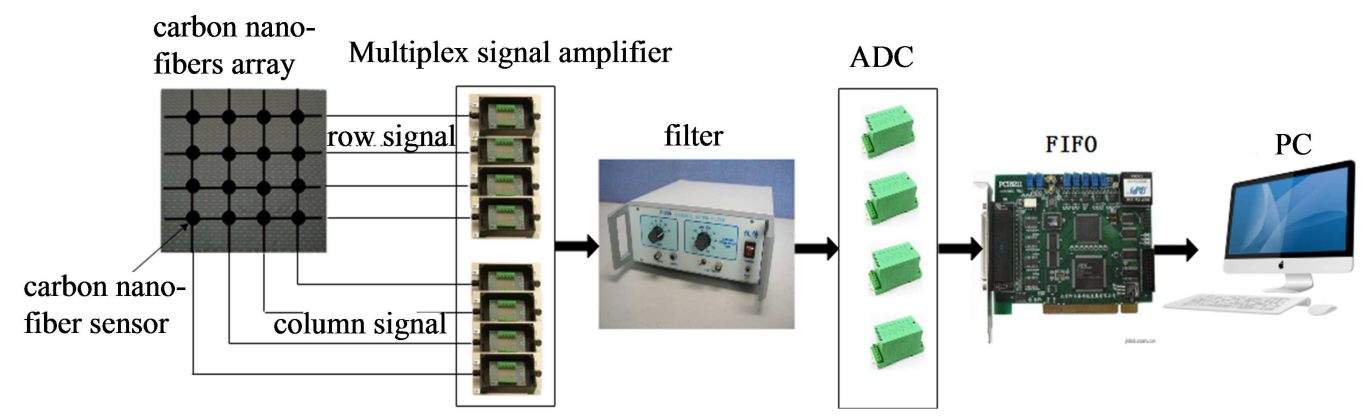

Figure 7. Damage location system structure of 3-D braided composite material based on carbon nano sensors.

The data collected by carbon nano-sensors were processed and analyzed using Wavelet and Singular Value Decomposition method for internal damage identification and location in specimen. First, using the two-dimensional wavelet threshold method to reduce noise, eliminate noise and extract useful signal [13]; Then, using the quaternary Singular Value Decomposition to extract the feature of the noise reduction data matrix. The shape and position of the specimen were determined by comparing the matrix singular value of the specimens with normal and no damage template. The specimens are shown in Figure 8, and The relevant parameters of the specimens are shown in Table 2. 
7 of 10

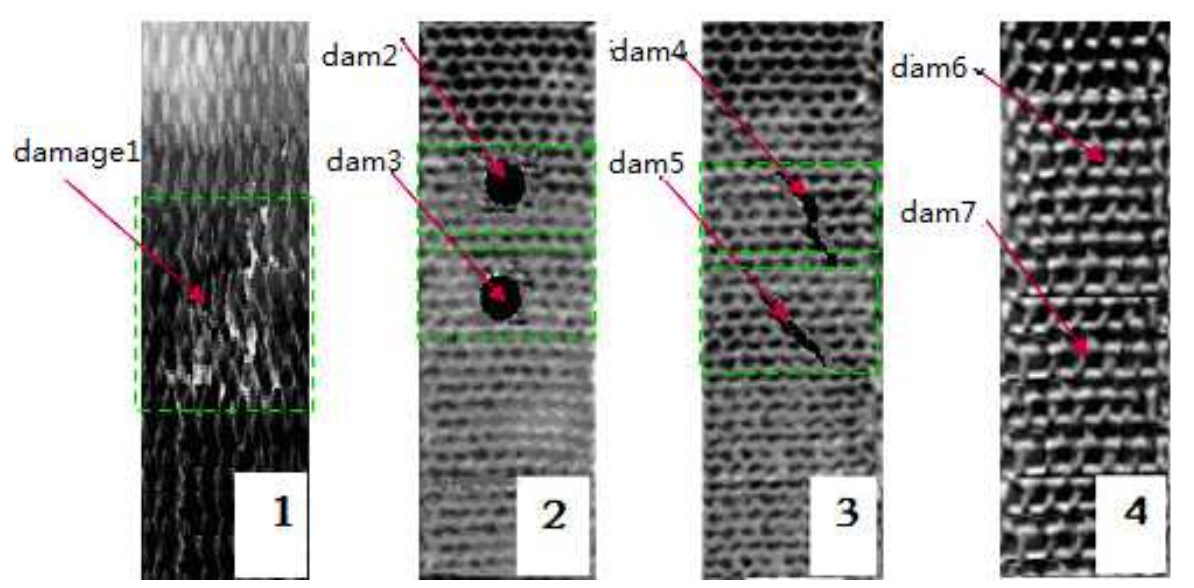

Figure 8. Specimens with known damage locations.

Table 2. specimens number and parameters.

\begin{tabular}{cccccc}
\hline Specimen number & $\mathbf{1}$ & $\mathbf{2}$ & $\mathbf{3}$ & $\mathbf{4}$ & $\mathbf{5}$ \\
\hline Density $\left(\mathrm{g} / \mathrm{cm}^{3}\right)$ & 1.5394 & 1.5486 & 1.4912 & 1.5185 & 1.5389 \\
Fiber volume fraction $(\%)$ & 58.84 & 62.38 & 45.40 & 56.65 & 60.30 \\
Braided angle $\left({ }^{\circ}\right)$ & 26.75 & 32.63 & 23.94 & 25.1 & 16.36 \\
Row space of carban nano-fibers $(\mathrm{mm})$ & 1.4 & 1.75 & 2.1 & 2.45 & 1.05 \\
Column space of carban nano-fibers $(\mathrm{mm})$ & 1.24 & 1.55 & 1.86 & 2.17 & 0.93 \\
Carbon nano-fiber sensor array size & $71^{*} 20$ & $57^{*} 16$ & $47^{*} 13$ & $40^{*} 11$ & $95^{*} 26$ \\
\hline
\end{tabular}

\section{Analysis of results}

According to Figure 7 experimental platform is set up, and column-direction resistance line obtained as shown in Figure 9. 

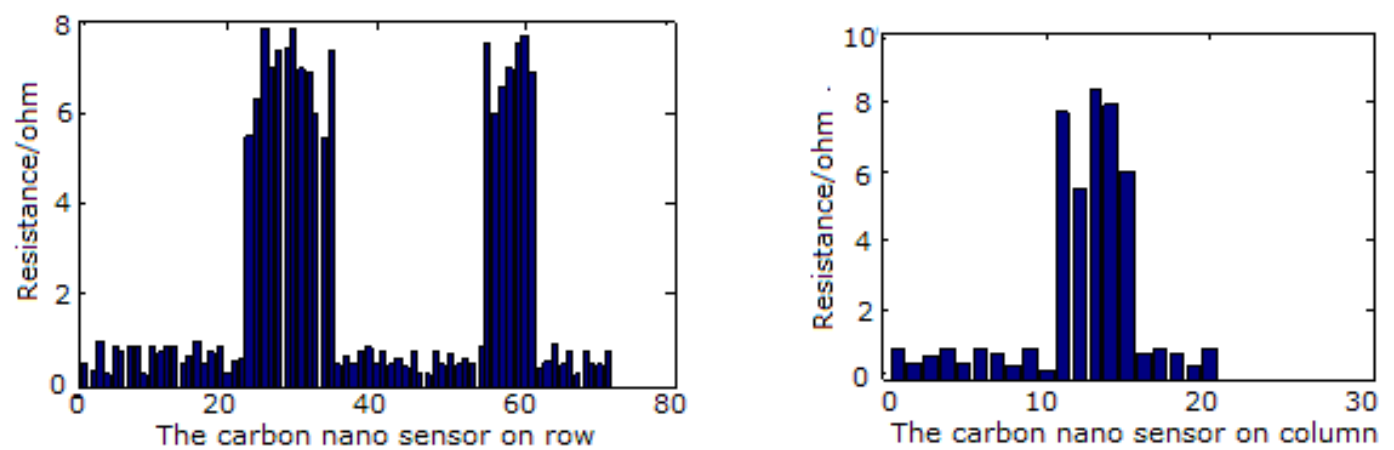

(a) The signals detected by carbon nano sensors in specimen 1
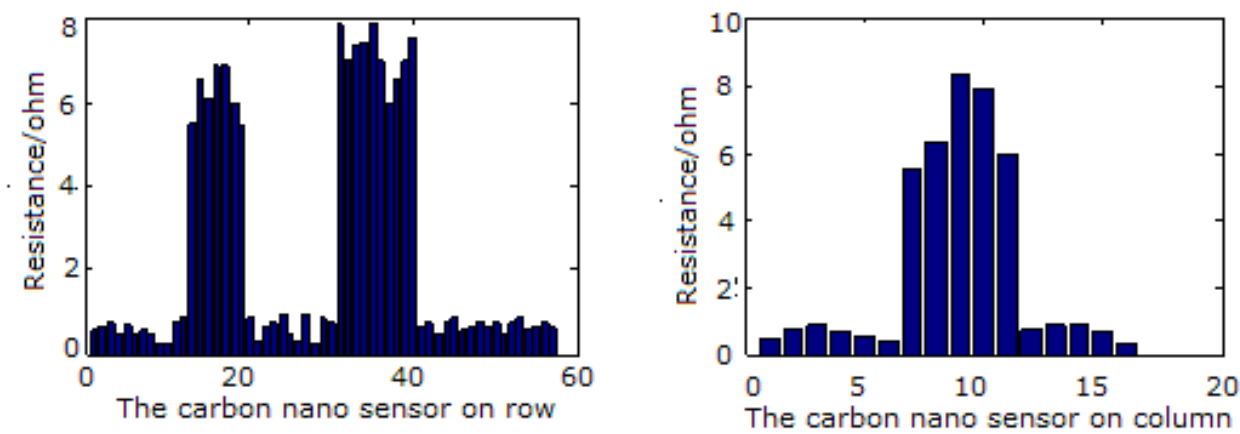

(b) The signals detected by carbon nano sensors in specimen 2
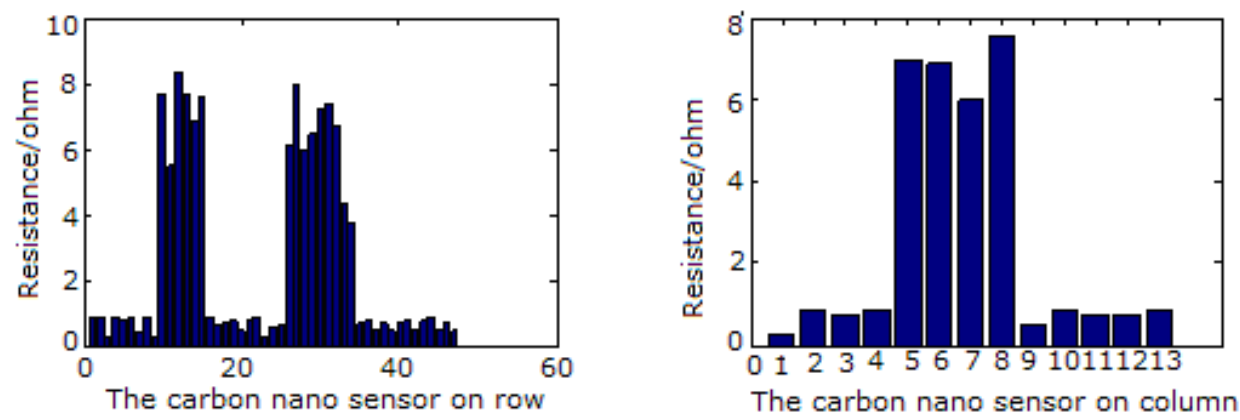

(c) The signals detected by carbon nano sensors in specimen 3
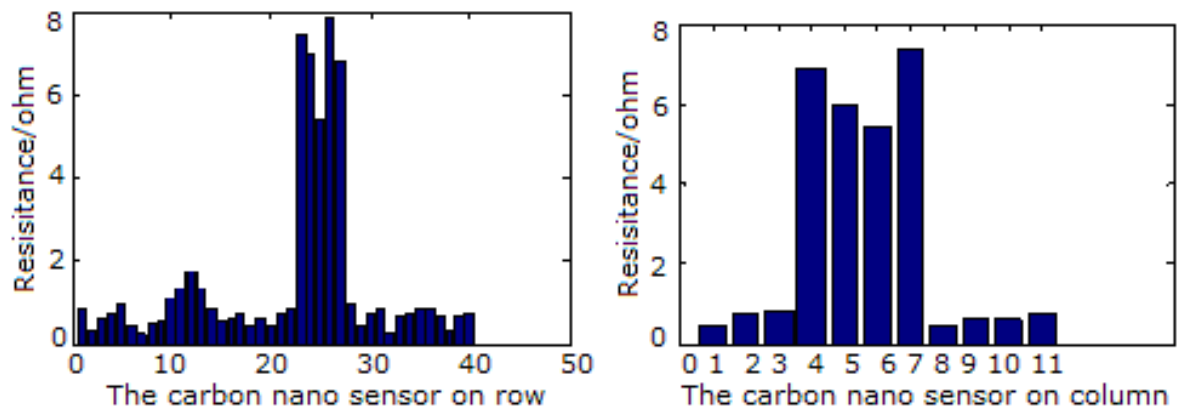

(d) The signals detected by carbon nano sensors in specimen 4
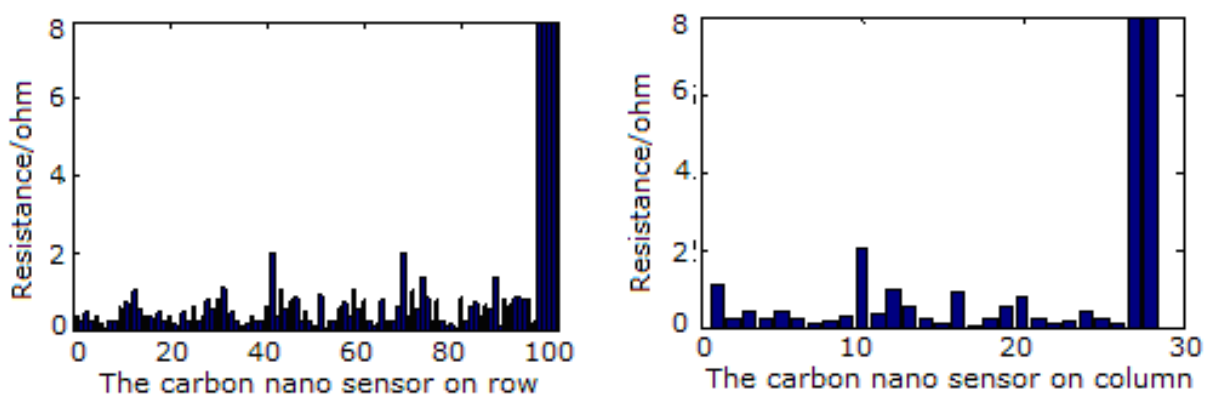

(e) The signals detected by carbon nano sensors in specimen 5

Figure 9. Abnormal data collected by carbon nano-sensor. 
In Figure 9 (d), the graph on the left shows the data mutation detected by the lateral carbon nano sensors, the graph on the right shows the data mutation detected by the vertical carbon nano sensors. As can be seen from graph on the left, the abnormal data mutation is more concentrated, which indicates that internal defects are concentrated. But in the graph on the right the abnormal data mutation is scattered which show that the longitudinal damage is sustained during load-carrying process of specimen.

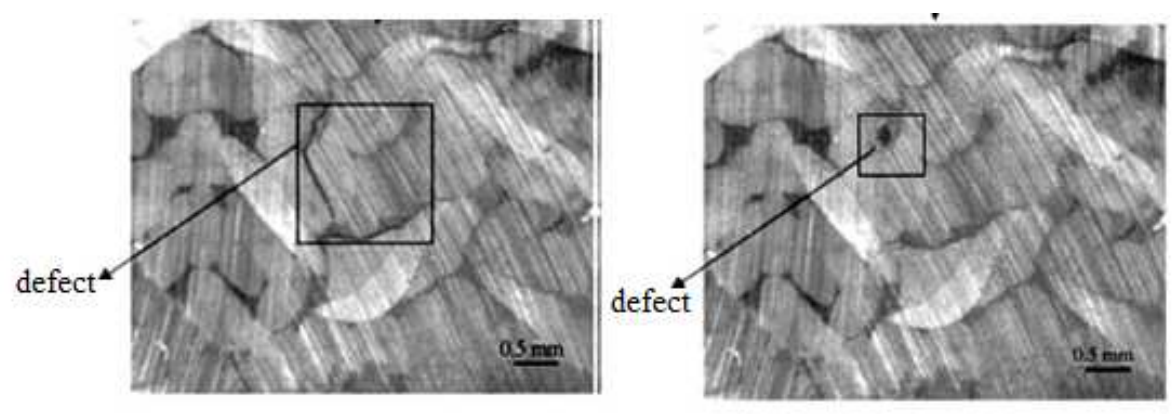

Figure 10. Section of the test piece.

Because of internal damage of the test piece makes strain even fracture occur in the embed carbon nano-fiber sensor, the resistance data measured have mutations. Therefore, according to the detection data flow abnormality of the composite material is abnormal[19], we can judge the existence of internal damage of the test piece.

\section{Conclusions}

In this paper, the carbon nano-fibers are made as sensors, carbon nano-fiber sensors are embedded in 3-D braided composite specimens by the 3-D six directional knitting technology. Through a specific algorithm, damage existence and damage location of 3-D braided composite specimens were monitored, and real-time data was unified processed. Therefor real time 3-D braided composite specimens damage state information is obtained. The following conclusions are obtained by experiments.

1. Compared to other sensors, carbon nano sensors used in health monitoring of three dimensional braided composite specimen has the advantage of easy embedded and carbon nanotubes has good linearity in the process of carrying.

2. Mechanical properties of three-dimensional braided composite specimen has benn little effected by carbon nano sensors embedded.

3. Built-in carbon nano-sensors can provide a new integrated and distributed sensor technology, and achieve the overall structure of the real-time health monitoring. Application of carbon nano sensor makes 3-D braided composite specimens health monitoring have good prospects for development.

Acknowledgments: Co-design of networked control systems under resource constraints. This work is supported by the National Natural Science Foundation of China (61403278)

Author Contributions: Q.D. and G.D. conceived and designed the experiments; J.C. performed the experiments; Q.D. analyzed the data; Q.D. wrote the paper.

Conflicts of Interest: The authors declare no conflict of interest.

\section{Abbreviations}

The following abbreviations are used in this manuscript: 


$\begin{array}{ll}\text { SVD } & \text { Singular Value Decomposition } \\ \text { CNT } & \text { Carbon Nano Tube } \\ \text { SCNT } & \text { Single Carbon Nano Tube } \\ \text { MCNT } & \text { Multi Carbon Nano Tube } \\ \text { CNF } & \text { Carbon Nano Fiber }\end{array}$

\section{References}

1. Wang X.M.; Xing Y.F. Developments in Research on 3D Braided Composites. ACTA AERONAUTICA ET ASTRONAUTICA SINICA. 2010, 31, 914-926.

2. Gu J.Y., Xu T.X, Yu R.B, Chen H.J. Study on Structural Health Monitoring and Its Key Technologies.Ordnance Industry Automation. 2011, 30, 61-64.

3. Wan Z.K.; Li J.D.jJia M.R. Structural health monitoring (SHM) of three-dimensional braided composite material using carbon nanotube thread sensors. Ordnance Industry Automation. 2013, 29, 616-621.

4. Xiao L.Y.; Kou X.F.; Zuo W.W.Study On Simulation of 3-D Braided Composites Weaving Process.Computer Engineering and Science. 2014, 36,719-724.

5. $\quad$ Lu S.W.; Feng C.L.;Nie P. Research progress of carbon nanotubes for J polymer composite health monitoring. Journal of aeronautical materials. 2015,35, 12-20.

6. Coleman J.N.; Khan U.; Blau W.J.Small but strong:a review of the mechanical properties of carbon nanotube-polymer composites.Carbon. 2006,44, 1624-1652.

7. Zhao H.B.; Zhang Y.Y.;Philip D. Carbon nanotube yarn strain sensors.Nanotechnology. 2010, 21, 305502-305507. 8. Jandro L.A.; Mark J.S.;Yi S.Novel distributed strain sensing in polymeric materials.Smart Mater, Struct. 2010, 19, 85007-86018.

9. Wu N.; Wang Q. Experimental studies on damage detection of beam structures with wavelet transform. International Journal of Engineering Science 2011, 49, 253-261.

10. Daniel I.M.; Luo J.J.;Schubel P.M. Three-dimensional characterization of textile composites. Composites Part B-Engineering 2008, 39, 13-19.

11. Chen L.; Li J.L.; Li X.M. Analysis of the law of motion of the yarn in the three dimensional knitting. Journal of composite materials. 2002,19, 71-74.

12. Tang D.F.; Qiao Z.W.; Fang K.P. Analysis of Mechanical Properties of 4-Step 3-D Braided Composites with Different Yarn Movement Styles. Fiber glass. 2017 2, 9-13.

13. Zhang D.; Zheng X.T.; Yang C. Damage tolerance property test of 3D braided composites. Acta Materiae Compositae Sinica. bf 2016, 33, 1048-1054.

14. Wu D.L. Three-cell model and 5-D braided structural composites. Composites Science and Technology. 1996, 56, 225-233.

15. Li X.Y.; Law S.S. Structural damage detection with statistical analysis from support excitation. Mechanical Systems and Signal Processing. 2008, 22, 1793-1808.

16. Rubinstein R.; Peleg T.; Elad M. Analysis K-SVD:A Dictionary-Learning Algorithm for the Analysis Sparse Model. IEEE TRANSACTIONS ON SIGNAL PROCESSING. 2013, 3.

17. Juneja A.K.; Rana B.; Agrawal R. K. A combination of singular value decomposition and multivariate feature selection method for diagnosis of schizophrenia using fMRI. BIOMEDICAL SIGNAL PROCESSING AND CONTROL. 2016, 27, 122-133.

18. Wan Z.K; Gong L.Y. Diagnosis research on health monitoring data of three-dimensional braided composite materials. Materials review. 2013, 27, 74-77. 